\title{
Saturation Throughput of IEEE 802.11 DCF in Multi-hop Ad Hoc Networks
}

\author{
Pan Li and Yuguang Fang \\ University of Florida, Gainesville, Florida 32611-6130 \\ Email: lipanleo@ufl.edu, fang@ece.ufl.edu
}

\begin{abstract}
IEEE 802.11 DCF has been widely used in wireless multi-hop ad hoc networks as the medium access control protocol. However, how well it can perform has not been well studied. In this paper, we first propose a new Markov chain model to analyze the per-node throughput by taking into consideration three important issues in multi-hop networks, i.e., physical carrier sensing threshold, signal-to-interference plus noise ratio threshold, and hidden terminal problems. Then, we validate our analytical model by simulations. We find the simulation results match our theoretical results well.
\end{abstract}

\section{INTRODUCTION}

Wireless ad hoc networks have drawn extensive attention due to the easy and quick deployment, as well as the low cost. They are playing important roles in both commercial and military applications. Since the wireless channel is shared by all the nodes in the network, a medium access control (MAC) protocol is needed to reduce collisions. Although initially standardized for wireless local area networks (WLANs), the IEEE 802.11 DCF (Distributed Coordination Function), known as Carrier Sense Multiple Access with Collision Avoidance (CSMA/CA), with an optional use of RTS/CTS [2], has been widely employed and becomes the de facto MAC protocol in ad hoc networks.

There are quite a number of papers in the literature on the performance of IEEE 802.11 MAC in WLANs, such as [3] [4] [7] [9]. Recently, some researches have extended the performance analysis of IEEE 802.11 MAC to multi-hop ad hoc networks. Wang et al. [13] propose a Markov chain to analyze the saturation throughput, which is the first analytical model in multi-hop ad hoc networks. By adopting Bianchi's result [3], Carvalho et al. [5] and Medepalli et al. [11] also study the performance of IEEE 802.11 MAC in multi-hop ad hoc networks. However, in [13], [5], and [11], physical carrier sensing threshold and signal-to-interference plus noise ratio (SINR) threshold are not considered,

978-1-4244-2677-5/08/\$25.00@) 2008 IEEE which are two important issues in IEEE 802.11 MAC. Specifically, a node can transmit packets only if the detected power level is below the physical carrier sensing threshold, and a transmission is successful only if the SINR at the receiver is above the SINR threshold. Although Durvy et al. [6] and Zhu et al. [15] take these two issues into consideration, they make too simple assumptions, i.e., 1) if an RTS transmission is successful in the first time slot, this RTS transmission would be successful, and 2) if an RTS transmission is successful, the following CTS, DATA, and ACK transmissions can all be successful. These assumptions are true in WLANs, but not necessarily true in multi-hop ad hoc networks due to the hidden terminal problems. Besides, Zhai et al. [14] and Kim et al. [8] study the performance of IEEE 802.11 MAC in multi-hop ad hoc networks, respectively. But, they do not consider the operation rules of IEEE 802.11 MAC, i.e., the four-way (RTS/CTS/DATA/ACK) handshake. Instead, they derive the achievable rates using Shannon Capacity formula, which as we all know, are much higher than what we can practically achieve in the networks.

In this paper, we analyze the per-node saturation throughput in multi-hop ad hoc networks using IEEE 802.11 MAC. We define saturation throughput as the throughput when all nodes in the network always have packets to send. The reason why we are interested in this issue is that in certain scenarios [10] [9], if the traffic arrival rate of each node is smaller than the saturation throughput, the queues of the nodes will have a proper, joint stationary distribution. In what follows, we use throughput instead of saturation throughput for simplicity. In our analysis, we propose a new Markov chain model to derive the per-node throughput. We differentiate the transmission range and the physical carrier sensing range to take physical carrier sensing threshold into consideration. We introduce two new concepts, namely hidden areas and hidden interfering areas, to take into account the hidden terminal problems and $S I N R$ threshold. Interestingly, we find that hidden interfering areas for RTS transmissions and for DATA 
transmissions are not necessarily the same, depending on the distance between a transmitter and a receiver, the physical carrier sensing threshold, and the SINR threshold. We also validate our analytical model by simulations, and the simulation results match our theoretical results well.

The rest of this paper is organized as follows. In Section II, we present our proposed Markov chain model to derive the per-node throughput. Simulations are carried out in Section III to verify our analytical model. We finally conclude this paper in Section IV.

\section{Throughput AnAlysis}

In this paper, we have the following assumptions on the node setting and placement:

- Nodes operate in time-slotted mode.

- Each node always has packets in its buffer to send.

- All nodes have the same physical carrier sensing range and the same transmission range, which are denoted by $R$ and $a R$, respectively, where $0<a \leq$ 1 .

- Nodes in the network are distributed according to two dimensional Poisson process with Poisson density $\rho(\rho>0)$. Thus, the probability that there are $i$ nodes in an area of $S$, denoted by $p(i, S)$, is

$$
p(i, S)=\frac{(\rho S)^{i}}{i !} e^{-\rho S} .
$$

Recall that in [3], Bianchi studies the performance of IEEE 802.11 DCF in wireless networks where any two nodes are within the transmission range of each other. Two hidden assumptions are made which are, first, when one node is transmitting RTS, this transmission can be successful only if none of the other nodes transmit in the first time slot of the RTS transmission, and second, the success of RTS transmission can ensure the successful four-way handshake (RTS/CTS/DATA/ACK). These two assumptions hold in single-hop ad hoc networks. However, they are not valid in multi-hop ad hoc networks due to hidden terminal problems.

Referring to Fig. 1, assume there is a transmission from node $A$ to node $\mathrm{B}$. The nodes in the shaded Area I are "hidden terminals" of node A, and we call this area "hidden area of transmitter $A$ ". Due to the hidden terminal problem, the successful transmission of RTS in the first slot cannot guarantee the success of this RTS transmission. Besides, since some of the hidden terminals may not be able to correctly receive the CTS from node B, the DATA transmissions could also be interrupted. As a result, the success of RTS transmission does not necessarily lead to a successful four-way handshake.

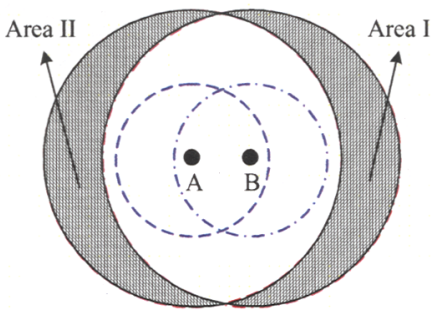

Fig. 1. Hidden area in multi-hop ad hoc networks. Small circles and large circles are transmission ranges and physical carrier sensing ranges, respectively.

On the other hand, we contend that CTS and ACK transmissions can be successful with a high probability. As shown in Fig. 1, when node A transmits an RTS, the nodes in A's transmission range will correctly receive this packet and keep silent for the rest of this whole transmission, i.e., a period of $3 * S I F S+T_{c t s}+T_{\text {data }}+T_{a c k}$, where $S I F S$ is the short interframe space as defined in IEEE 802.11 [2], $T_{c t s}, T_{d a t a}$, and $T_{a c k}$ are the transmission time of CTS, DATA, and ACK frames, respectively. Moreover, the nodes outside A's transmission range but within A's physical carrier sensing range cannot decode the RTS and will keep silent for a period of EIFS, which is equal to $S I F S+T_{c t s}+D I F S$. Thus, the nodes in Area II will not interrupt node A's CTS reception. In other words, there is no hidden terminal problem for CTS reception. Similarly, these nodes will not interrupt node A's ACK reception, either. Although node A is still interfered by those nodes outside its physical carrier sensing range when receiving $\mathrm{CTS}$ or $\mathrm{ACK}$, the interference caused is very limited. Therefore, we consider CTS and ACK transmissions always successful in this paper.

Based on the analysis above, we can model the states of a node in the network by a Markov chain. As shown in Fig. 2, an observed node has four states:

- "Waiting" is the state when the node backs off or defers its transmissions for other nodes.

- "RTS collision" is the state when the node initiates a RTS transmission but fails.

- "DATA collision" is the state when the node initiates a DATA transmission but fails, which means the RTS/CTS handshake before DATA transmission is successful.

- "Success" is the state when the node finishes the four way handshake successfully.

Let $\pi_{w}, \pi_{r}, \pi_{d}$, and $\pi_{s}$ denote the steady-state probabilities of states Waiting, RTS collision, DATA collision, and Success, respectively. Then, the corresponding time periods for each state, denoted by $T_{w}, T_{r}, T_{d}$, and $T_{s}$, 


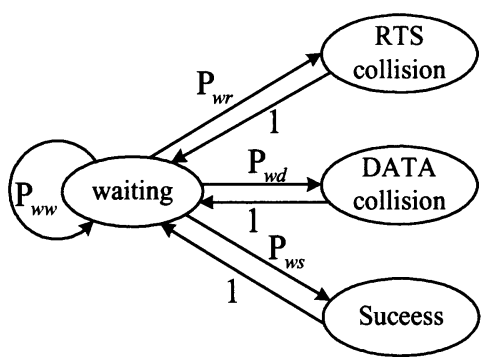

Fig. 2. Node state transition diagram considering hidden terminals.

respectively, are

$$
\begin{aligned}
T_{w}= & \delta \\
T_{r}= & \frac{L_{r t s}}{r_{\text {basic }}}+E I F S \\
T_{d}= & \frac{L_{r t s}+L_{c t s}}{r_{\text {basic }}}+\left(\frac{L_{H P H Y}+L_{H M A C}}{r_{\text {basic }}}+\frac{L_{\text {data }}}{r_{\text {data }}}\right) \\
& +E I F S+2 * S I F S \\
T_{s}= & \frac{L_{r t s}+L_{c t s}+L_{a c k}}{r_{\text {basic }}}+3 * S I F S \\
& +\left(\frac{L_{H P H Y}+L_{H M A C}}{r_{\text {basic }}}+\frac{L_{\text {data }}}{r_{\text {data }}}\right)
\end{aligned}
$$

where $\delta$ is the period of a single time slot, $L_{r t s}, L_{c t s}$, $L_{\text {data }}$, and $L_{a c k}$ are the length of RTS, CTS, DATA, and ACK frames, respectively, $L_{H P H Y}$ and $L_{H M A C}$ are the length of physical and MAC headers of a DATA frame, respectively, $r_{b a s i c}$ and $r_{d a t a}$ are the basic rate and the data rate, respectively, EIFS and SIFS are as defined in IEEE 802.11 standard [2].

Thus, the per-node throughput, denoted by $\lambda$, can be calculated as follows:

$$
\lambda=\frac{\pi_{s} \cdot L_{d a t a}}{\pi_{w} T_{w}+\pi_{r} T_{r}+\pi_{d} T_{d}+\pi_{s} T_{s}} .
$$

Obviously, in order to derive the per-node throughput, we need to first obtain the four steady-state probabilities.

Denote by $P_{w w}, P_{w r}, P_{w d}$, and $P_{w s}$ the transition probabilities from Waiting to Waiting, from Waiting to RTS collision, from Waiting to DATA collision, and from Waiting to Success, respectively. We have

$$
\begin{aligned}
\pi_{w} & =\pi_{w} P_{w w}+\pi_{r}+\pi_{d}+\pi_{s} \\
1 & =\pi_{w}+\pi_{r}+\pi_{d}+\pi_{s}
\end{aligned}
$$

which results in

$$
\pi_{w}=\frac{1}{2-P_{w w}} .
$$

Since $P_{w w}$ is the probability that a node continues to stay in Waiting state,

$$
P_{w w}=P_{w w}^{1} \cdot P_{w w}^{2}
$$

where $P_{w w}^{1}$ is the probability that the observed node does not transmit, and $P_{w w}^{2}$ is the probability that none of the nodes in the observed node's carrier sensing range transmit, i.e.,

$$
\begin{aligned}
P_{w w}^{1} & =1-\tau \\
P_{w w}^{2} & =\sum_{i=0}^{\infty}(1-\tau)^{i} \frac{\left(\rho \pi R^{2}\right)^{i}}{i !} e^{-\rho \pi R^{2}} \\
& =e^{-\tau N}
\end{aligned}
$$

where $N=\rho \pi R^{2}$ is the expected number of neighbors in one node's carrier sensing range.

Substituting (8) and (9) into (7), and then into (6), we obtain

$$
\pi_{w}=\frac{1}{2-(1-\tau) e^{-\tau N}}
$$

Next, we attempt to derive the steady-state probability $\pi_{s}$. Since

$$
\pi_{s}=P_{w s} \cdot \pi_{w},
$$

we need to find the transition probability $P_{w s}$. Define $P_{w s}(r)$ as the probability that a transmitter can finish a four-way handshake successfully under the condition that the transmitter and its corresponding receiver are at a distance $r$ away from each other, where $0<r \leq a R$. Then,

$$
P_{w s}(r)=P_{0} \cdot P_{1} \cdot P_{2} \cdot P_{3}(r) \cdot P_{4}(r) \cdot P_{5}(r)
$$

where

$P_{0}=\operatorname{Pr}\{$ Transmitter transmits in a time slot $\}$

$P_{1}=\operatorname{Pr}\{$ Receiver does not transmit in the same time slot\}

$P_{2}=\operatorname{Pr}\{$ None of the nodes in the sensing range of the transmitter transmit in the same time slot\}

$P_{3}(r)=\operatorname{Pr}\{$ None of the nodes in the hidden area of the transmitter transmit in $\left\lceil\frac{L_{r t s}}{r_{\text {basic }} \delta}\right\rceil$ slots $\left.\mid r\right\}$

$P_{4}(r)=\operatorname{Pr}$ \{No node in the hidden interfering area of the receiver transmits in $\left\lceil\frac{L_{r t s}}{r_{b a s i c} \delta}\right\rceil$ slots $\left.\mid r\right\}$

$P_{5}(r)=\operatorname{Pr}$ \{No node in the hidden interfering area of the receiver transmits in $\left\lceil\left(\frac{L_{\text {data }}}{r_{\text {data }}}-E I F S\right) / \delta\right\rceil$ slots $\left.\mid r\right\}$

Denote the hidden interfering area of a receiver by $S_{H I}$, which is defined as follows:

$$
S_{H I}=\bar{S}_{C S} \cap S_{I},
$$

with $S_{C S}$ the physical carrier sensing area of the corresponding transmitter, $\bar{S}_{C S}$ the area outside the physical carrier sensing range of the transmitter, and $S_{I}$ the 


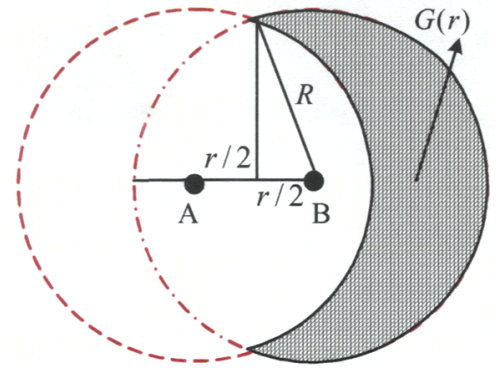

Fig. 3. Hidden areas of the transmitter.

interfering area of the receiver where a single node's transmission can ruin the receiver's reception. Obviously,

$$
S_{C S}=\pi R^{2}, \quad S_{I}=\pi r_{I}^{2} .
$$

where $r_{I}$ is the interference range.

Recall the power propagation model given in [12]:

$$
P_{r}(d)=P_{t} \frac{C}{d^{\gamma}},
$$

where $P_{t}$ and $P_{r}$ are the transmitted power and the received power, respectively, $C$ is a constant related to the antenna profiles of the transmitter and the receiver, wavelength, and so on, $d$ is the distance between the transmitter and the receiver, and $\gamma$ is the path loss exponent. Let $S I N R$ denote the Signal-to-Interference plus Noise Ratio required to correctly receive a signal. We have

$$
\frac{P_{t} C / r^{\gamma}}{P_{t} C / r_{I}^{\gamma}}=S I N R
$$

i.e.,

$$
r_{I}=r \cdot S I N R^{\frac{1}{\gamma}} .
$$

In (12), $P_{3}(r), P_{4}(r)$, and $P_{5}(r)$ are all functions of $r$. $P_{3}(r)$ can be interpreted as that all the nodes within the receiver's carrier sensing range should keep silent during a period of $\left\lceil\frac{L_{r t s}}{r_{\text {basic }} \delta}\right\rceil$ slots before an RTS is transmitted since otherwise the receiver cannot receive the RTS due to its physical carrier sensing. Since the transmitter was in Waiting state, we only need the hidden terminals of the transmitter to keep silent. $P_{4}(r)$ can be interpreted as that the vulnerable period for the RTS transmission is only $\left\lceil\frac{L_{r t s}}{r_{\text {basic }} \delta}\right\rceil$ slots during which those hidden interfering terminals should keep silent. $P_{5}(r)$ can be interpreted as that the DATA transmission can be interrupted because those nodes in the hidden interfering area of the receiver did not correctly receive the CTS from the receiver. The nodes in the hidden interfering area of the receiver will keep silent for a period of EIFS during the DATA transmission, and thus the the vulnerable period for DATA transmission is $\left\lceil\left(\frac{L_{d a t a}}{r_{d a t a}}-E I F S\right) / \delta\right\rceil$ slots.
From the above, we can obtain

$$
\begin{aligned}
& P_{0}=\tau \\
& P_{1}=1-\tau \\
& P_{2}=P_{w w}^{2}=e^{-\tau N} \\
& P_{3}(r)=\left[\sum_{i=0}^{\infty}(1-\tau)^{i} \frac{(\rho G(r))^{i}}{i !} e^{-\rho G(r)}\right]^{\left\lceil\frac{L_{r t s}}{r_{b a s i c} \delta}\right]} \\
& =e^{-\tau \rho G(r)\left\lceil\frac{L_{r t s}}{r_{\text {basic }} \delta}\right\rceil} \\
& P_{4}(r)=\left[\sum_{i=0}^{\infty}(1-\tau)^{i} \frac{(\rho H(r))^{i}}{i !} e^{-\rho H(r)}\right]^{\left\lceil\frac{L_{r t s}}{r_{b a s i c^{\delta}}}\right\rceil} \\
& =e^{-\tau \rho H(r)\left\lceil\frac{L_{r t s}}{r_{\text {basic }} \delta}\right\rceil} \\
& =e^{-\tau \rho H^{\prime}(r)\left\lceil\left(\frac{L_{\text {data }}}{r_{\text {data }}}-E I F S\right) / \delta\right\rceil},
\end{aligned}
$$

where $G(r)$ is the hidden area of the transmitter, $H(r)$ and $H^{\prime}(r)$ are the hidden interfering areas for RTS and DATA transmissions, respectively.

As shown in Fig. 3, we have

$$
\begin{aligned}
G(r) & =\pi R^{2}-4\left[\frac{1}{2} R^{2} \arccos \left(\frac{r}{2 R}\right)-\frac{r}{4} \sqrt{R^{2}-\frac{r^{2}}{4}}\right] \\
& =\pi R^{2}-2 R^{2} \arccos \left(\frac{r}{2 R}\right)+r \sqrt{R^{2}-\frac{r^{2}}{4}}(20)
\end{aligned}
$$

To calculate $H(r)$, we need to consider three cases:

1) $R \geq r_{I}+r$, i.e., $r \leq \frac{R}{\operatorname{SINR}^{\frac{1}{\gamma}}+1}$. This case is shown in Fig. 4(a), and

$$
H(r)=0 .
$$

2) $r_{I}-r<R<r_{I}+r$, i.e., $\frac{R}{\operatorname{SIN} R^{\frac{1}{\gamma}}+1}<r<$ $\frac{R}{S I N R^{\frac{1}{\gamma}}-1}$. This case is as shown in Fig. 4(b), and

$$
H(r)=r_{I}^{2} \beta+r R \sin \alpha-R^{2} \alpha,
$$

where

$$
\begin{aligned}
\alpha & =\arccos \frac{r^{2}+R^{2}-r_{I}^{2}}{2 r R} \\
\beta & =\pi-\arccos \frac{r^{2}+r_{I}^{2}-R^{2}}{2 r r_{I}} .
\end{aligned}
$$

3) $R \leq r_{I}-r$, i.e., $r \geq \frac{R}{S I N R^{\frac{1}{\gamma}}-1}$. This case is shown in Fig. 4(c), and

$$
H(r)=\pi r_{I}^{2}-\pi R^{2} .
$$

Recall that $H^{\prime}(r)$ denotes the hidden interfering area for DATA transmissions. Since the CTS transmission has been successful, those nodes within the receiver's transmission range will keep silent for the remaining time of the current transmission, and will not interfere 

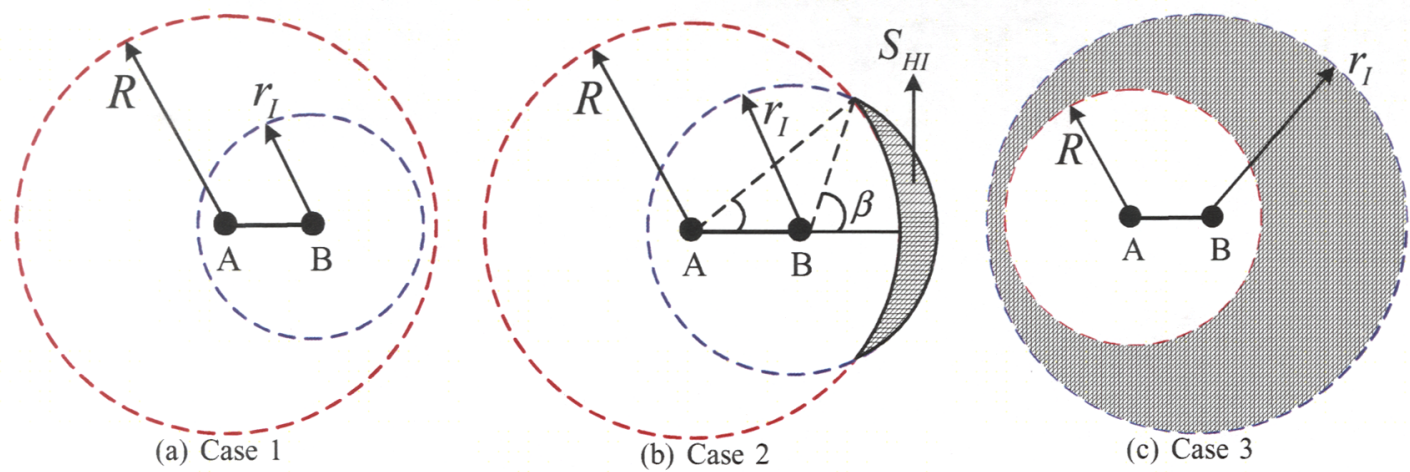

(c) Case 3

Fig. 4. Hidden interfering areas for RTS transmission.

with the receiver's DATA reception. Let $S_{T}$ denote the transmission area of the receiver. We have

$$
H^{\prime}(r)=H(r) \backslash\left(H(r) \cap S_{T}\right) .
$$

Define $S_{T I}$ as $H(r) \cap S_{T}$. We need to consider two cases to calculate $S_{T I}$ :

1) $2 a R \leq R$, i.e., $a \leq \frac{1}{2}$, as shown in Fig. 5(a). We have

$$
S_{T I}=0 .
$$

2) $2 a R>R$, i.e., $\frac{1}{2}<a \leq 1$, as shown in Fig. 5(b). We have

$$
S_{T I}=\left\{\begin{array}{lr}
0 & \text { if } r \leq(1-a) R \\
r_{I}^{2} \beta^{\prime}+r R \sin \alpha^{\prime}-R^{2} \alpha^{\prime} \text { if } r>(1-a) R
\end{array}\right.
$$

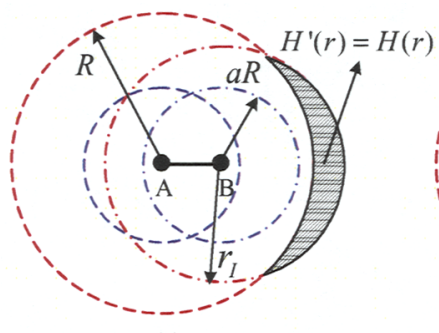

(a)

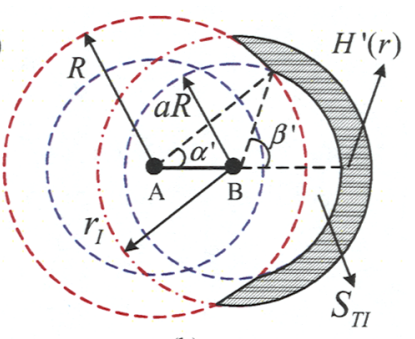

(b)

Fig. 5. Hidden interfering areas for DATA transmission.

where

$$
\begin{aligned}
P_{w d} & =\int_{0}^{a R} \frac{2 r}{a^{2} R^{2}} P_{w d}(r) d r \\
P_{w d}(r) & =P_{0} \cdot P_{1} \cdot P_{2} \cdot P_{3}(r) \cdot P_{4}(r) \cdot\left(1-P_{5}(r)\right)
\end{aligned}
$$

Finally, the steady-state probability $\pi_{r}$ is

$$
\pi_{r}=1-\pi_{w}-\pi_{d}-\pi_{s} .
$$

Up to now, we have successfully derived all the four steady-state probabilities. Substituting them into (5), we can eventually obtain the per-node throughput.

\section{Simulation Results}

In this section, we carry out simulations to validate our theoretical model. We use NS2 (Version 2.29 [1]) as the network simulator. The simulation parameters are shown in Table I.

Besides, as shown in Fig. 6, we use a grid topology to perform the simulation, where 49 nodes are uniformly distributed in 7 rows and 7 columns. The distances between two neighboring rows and two neighboring columns are both 250 meters.

Since in the simulation the nodes are uniformly distributed, (16), (17), (18), and (19) should instead be (1$\tau)^{N},(1-\tau)^{\rho G(r)\left\lceil\frac{L_{r t s}}{r_{b a s i c} \delta}\right.},(1-\tau)^{\rho H(r)\left\lceil\frac{L_{r t s}}{r_{\text {basic }} \delta}\right.}$, and $(1-$ $\tau)^{\rho H^{\prime}(r)\left\lceil\left(\frac{L_{\text {data }}}{r_{\text {data }}}-E I F S\right) / \delta\right\rceil}$, respectively. However, according to the simulation settings, $N$ in (16) is equal to 13 ,

By substituting (22) into (11), we can obtain $\pi_{s}$.

Similarly, the steady-state probability $\pi_{d}$ can be obtained by

$$
\pi_{d}=P_{w d} \cdot \pi_{w}
$$


TABLE I

SOME CONFIGURATION PARAMETERS.

\begin{tabular}{|l|l|}
\hline Parameters & Value \\
\hline Path loss exponent & 4 \\
Data rate & $1 \mathrm{Mbps}$ \\
Basic rate & $1 \mathrm{Mbps}$ \\
Payload & $512 \mathrm{bytes}$ \\
SIFS & $10 \mu \mathrm{s}$ \\
DIFS & $50 \mu \mathrm{s}$ \\
Backoff slot & $20 \mu \mathrm{s}$ \\
MAC Header & $272 \mathrm{bits}$ \\
PHY Header & $192 \mathrm{bits}$ \\
RTS & $160 \mathrm{bits}+$ PHY Header \\
CTS & $112 \mathrm{bits}+$ PHY Header \\
ACK & $112 \mathrm{bits}+$ PHY Header \\
SINR & $10 \mathrm{~dB}$ \\
Transmission range & $250 \mathrm{~m}$ \\
Physical carrier sensing range & $550 \mathrm{~m}$ \\
\hline
\end{tabular}

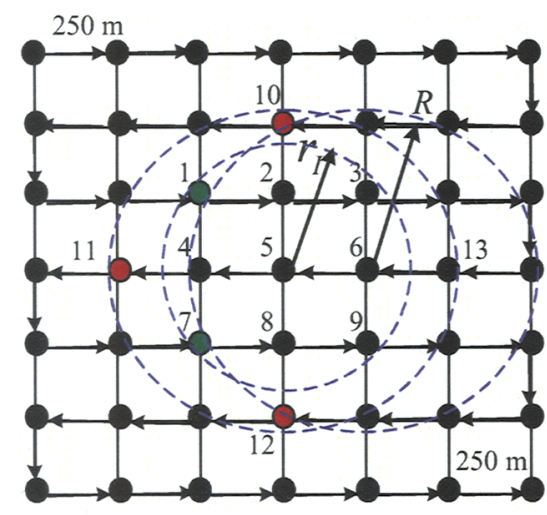

Fig. 6. A grid topology.

and hence $(1-\tau)^{N}$ can be well approximated by $e^{-\tau N}$. Similarly, in the simulation, $\rho G(r), \rho H(r)$, and $\rho H^{\prime}(r)$ in (17), (18), and (19), are all bigger than $1,\left\lceil\frac{L_{r t s}}{r_{\text {basic }} \delta}\right\rceil$ in (17) and (18) is equal to 18 , and $\left\lceil\left(\frac{L_{d a t a}}{r_{\text {data }}}-E I F S\right) / \delta\right\rceil$ in (19) is equal to 228 , and hence $(1-\tau)^{\rho G(r)\left\lceil\frac{L_{r t s}}{r_{b a s i c} \delta}\right.}$, $(1-\tau)^{\rho H(r)\left\lceil\frac{L_{r t s}}{r_{\text {basic }} \delta^{\delta}}\right.}$, and $(1-\tau)^{\rho H^{\prime}(r)\left\lceil\left(\frac{L_{d a t a}}{r_{\text {data }}}-E I F S\right) / \delta\right\rceil}$ can also be well approximated by (17), (18), and (19), respectively. Thus, we conclude that we can use these simulation settings to well evaluate the performance of our theoretical results obtained before.

We carry out multi-hop communications in the grid network. Specifically, we set up 49 CBR traffic flows, one for each node. The destination nodes of those flows are randomly chosen. AODV (Ad Hoc OnDemand Distance Vector) is selected as the routing protocol. We collect the average transmission probability and the average throughput over the nine transmitting nodes in the center of the grid, i.e., nodes 1-9. We repeat this process ten times, and compare the simulation result with the theoretical result. Note that according to our theoretical model, nodes 1,4 , and 7 have 4 hidden

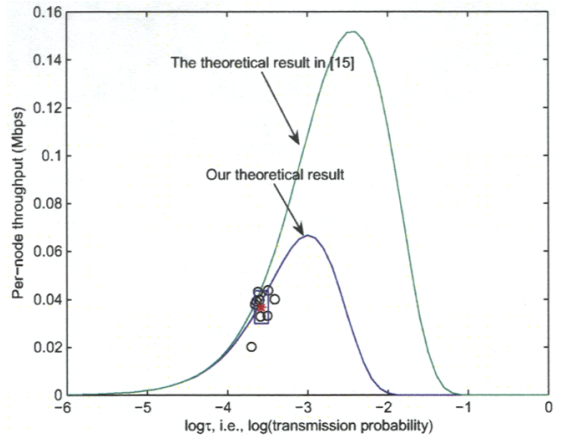

Fig. 7. Theoretical and simulation results for nodes 1-9 when $C W \min =31, C W \max =1023$.

terminals, and 2 hidden interfering nodes for both RTS and DATA transmissions, and nodes $2,3,5,6,8$, and 9 have 5 hidden terminals, and 2 hidden interfering nodes for both RTS and DATA transmissions. Thus, on average, each of the nine center nodes has $14 / 3$ hidden terminals, and 2 hidden interfering nodes for both RTS and DATA transmissions, i.e., $\rho G(r)=14 / 3$, and $\rho H(r)=\rho H^{\prime}(r)=2$.

We depict the results for $C W \min =31, C W \max =$ 1023, and for $C W \min =C W \max =31$, in Fig. 7 and Fig. 8, respectively. The small rectangles indicates the variances of the transmission probability and throughput. When $C W \min =31, C W \max =1023$, the pernode throughput in the simulation results range from $65 \%$ to $126 \%$ of that in our theoretical results. The average per-node throughput in the simulation results over the ten runs is about $93 \%$ of that in our theoretical result, and about $84 \%$ of that in the theoretical result in [15], respectively. The variance of $\log$ (transmission probability) and of the throughput are 0.089 and 0.0071 , respectively. When $C W \min =C W \max =31$, the pernode throughput in the simulation results range from $80 \%$ to $98 \%$ of that in our theoretical results. The average per-node throughput in the simulation result over the ten runs is about $88 \%$ of that in our theoretical result, and about $77 \%$ of that in the theoretical result in [15], respectively. The variance of $\log$ (transmission probability) and of the throughput are 0.062 and 0.0034 , respectively. We find that in both cases our theoretical results match the simulation results well, and the theoretical results in [15] are much higher than simulation results. We also observe that when $C W \min$ is equal to $C W \max$, the network can provide better fairness.

\section{CONCLUSION}

In this paper, we derive analytical results on the pernode saturation throughput in multi-hop ad hoc networks using IEEE 802.11 DCF. Simulations are carried out 


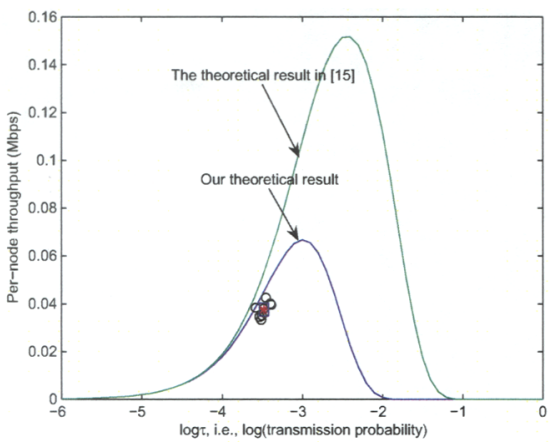

Fig. 8. Theoretical and simulation results for nodes 1-9 when $C W \min =C W \max =31$.

where the simulation results well match our theoretical results.

\section{ACKNOWLEDGMENT}

This work was supported in part by U.S. National Science Foundation (NSF) under grant CNS-0721744 and grant DBI-0529012.

\section{REFERENCES}

[1] http://www.isi.edu/nsnam/ns/.

[2] Wireless lan medium access control (mac) and physical layer (phy) specifications. IEEE Standards Working Group, 1999.

[3] G. Bianchi. Performance analysis of the ieee 802.11 distributed coordination function. IEEE Journal on Selected Areas in Communications, 18(3):535-547, March 2000.

[4] F. Cali, M. Conti, and E. Gregori. Dynamic tuning of the ieee 802.11 protocol to achieve a theoretical throughput limit. IEEE/ACM Transactions on Networking, 8(6):785-799, December 2000 .

[5] M. Carvalho and J. Garcia-Luna-Aceves. A scalable model for channel access protocols in multihop ad hoc networks. In Proceeding of ACM MobiCom, Philadelphia, PA, USA, September 2004.

[6] M. Durvy, O. Dousse, and P. Thiran. Modeling the 802.11 protocol under different capture and sensing capabilities. In Proceeding of IEEE INFOCOM Miniconference, Anchorage, AK, USA, May 2007.

[7] Z. Hadzi-Velkov and L. Gavrilovska. Performance of the ieee 802.11 wireless lans under influence of hidden terminals and pareto distributed packet traffic. In Proceedings of IEEE PWCS'99, February 1999.

[8] A. Khandani, J. Abounadi, E. Modiano, and L. Zhang. Cooperative routing in wireless networks. In Proceedings of Allerton Conference on Communications, Control and Computing, October 2003.

[9] A. Kumar, E. Altman, D. Miorandi, and M. Goyal. New insights from a fixed point analysis of single cell ieee 802.11 wlans. In Proceeding of the IEEE International Conference on Computer Communications (INFOCOM'03), Miami, FL, USA, March 2005.

[10] A. Kumar and D. Patil. Stability and throughput analysis of unslotted cdma-aloha with finite number of users and code sharing. Telecommunication Systems, 8:257-275, 1997.
[11] K. Medepalli and F. Tobagi. Towards performance modeling of ieee 802.11 based wireless networks: A unified framework and its applications. In Proceeding of the IEEE International Conference on Computer Communications (INFOCOM'06), Barcelona, Spain, April 2006.

[12] T. Rappaport. Wireless Communications: Principles and Practice (Second Edition). Prentice-Hall PTR, 2002.

[13] Y. Wang and J. Garcia-Luna-Aceves. Performance of collision avoidance protocols in single-channel ad hoc networks. In Proceedings of the 10th IEEE International Conference on Network Protocols (ICNP), Paris, France, November 2002.

[14] H. Zhai and Y. Fang. Physical carrier sensing and spatial reuse in multirate and multihop wireless ad hoc networks. In Proceeding of the IEEE International Conference on Computer Communications (INFOCOM'06), Barcelona, Spain, April 2006.

[15] Y. Zhu, Q. Zhang, Z. Niu, and J. Zhu. On optimal physical carrier sensing: Theoretical analysis and protocol design. In Proceeding of IEEE INFOCOM Miniconference, Anchorage, AK, USA, May 2007. 